\title{
Al Tools Boost Simple Technologies in a Shared World
}

\author{
Bicycles and indoor lighting are among many everyday features that can \\ benefit from recent advances in artificial intelligence.
}

\section{By Rachel Berkowitz}

$\mathrm{n}$ the digital world, the stuff of science fiction has become
the fabric of daily life: Our environments tailor themselves
to our personal tastes; our handheld devices infer our thoughts; and machines perform many daily tasks at home and at work. Earlier this month at the Future Technologies Conference (FTC) 2020, researchers from around the world came together virtually to discuss the latest computing, engineering, and data science behind artificial intelligence tools. Motivating some of these developments is the growing need for thoughtful and safe sharing of spaces, from cities to workplaces. Representative of this sharing emphasis are two novel concepts: bicycles rigged for data collection and office environments that put everyone in the spotlight.

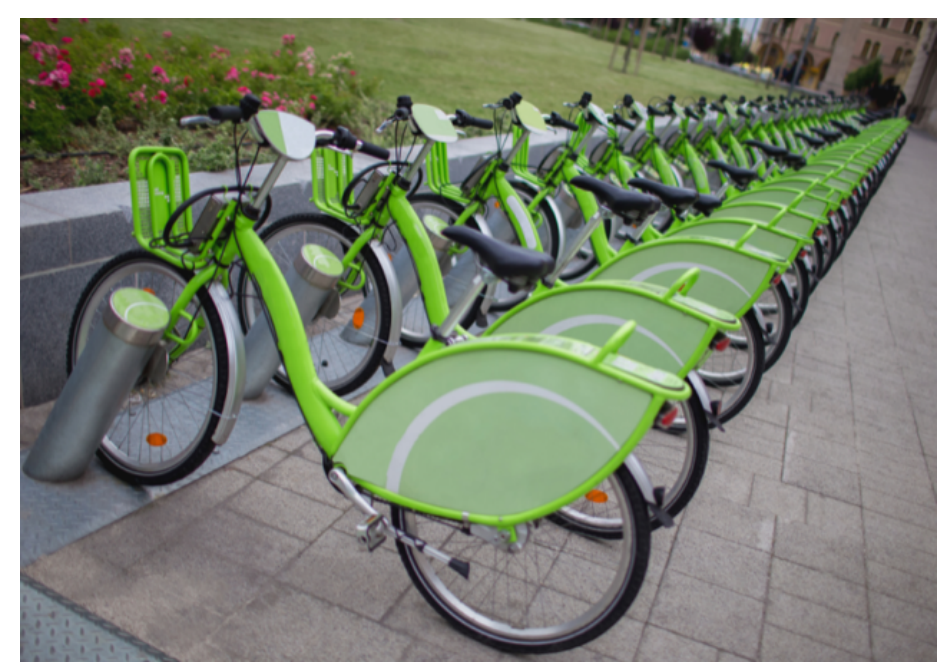

Bike-sharing fleets could become roving data collectors. Credit: Visual Intermezzo
Bicycles may be tried-and-true technology, but they still have room for innovation. As bicycle sharing becomes a popular solution for city dwellers, some researchers are seeing an opportunity to create decentralized networks of mobile sensors. Several bike-sharing companies have started to equip their vehicles with detectors that collect information about environmental and geospatial conditions, which urban planners can use to improve city infrastructure and other community development projects.

To harness the potential of these new data streams, Andres Rico and his colleagues at the Massachusetts Institute of Technology (MIT) have designed a mobile platform for electric-assist bicycles that incorporates a camera, a GPS module, and multiple environmental sensors. Data collected by each part of the system are combined to give a holistic description of a given bike trip and a better understanding of the rider's relationship with the bike and the immediate environment. This, in turn, leads to deeper understanding of the urban surroundings. For instance, it can identify commuter route preferences that may indicate riders' perceptions of safety in their community.

Rico and his colleagues have developed software that can uncover more subtle information within bike trip measurements. For example, by examining the location of frequent changes in acceleration they could identify hazardous road conditions, such as potholes or dangerous intersections. The software could also utilize bicycle-provided readings of temperature, humidity, light intensity, and bike speed to pinpoint areas that are adjacent to heavy car traffic, especially high-speed traffic that could pose a danger. The team have met with city officials in Boston and Shibuya, Japan, where, says Rico, there is plenty of interest in supporting further 
development of the system.

Another aspect of shared living addressed at FTC is lighting. Smart lighting systems respond to voice and gestures to illuminate areas for particular users. However, this green solution can pose a problem in a communal workspace, where different workers may have different lighting needs. To create a more user-friendly space, Elena Kodama and her MIT colleagues developed a dynamic system that senses occupants' activity and accordingly generates a separately controlled light for each person in a room.

According to Kodama, previous research on smart shared lighting has mainly focused on energy-saving solutions that turn off lights when no one is around. Her team's approach brings in context awareness, which is a programming strategy that uses data from the environment to form a tailored response. By implementing several advanced control features for programmable light fixtures, the MIT team developed a prototype that compares real-time user position data to the lighting locations. Based on that mapping, commands are sent to adjust the brightness or change the color temperature of the corresponding light fixtures.

Kodama's system can follow up to six users around the room and turn lights out when all the users leave. Hand gestures can change the hue, and the light can be equally split based on the number of users present in the shared space. When two or more users stand close to each other, features of their associated lights blend according to an algorithm that favors the stronger light. As the system becomes context aware, it could eventually learn from each user's behavior to anticipate their lighting requirements.

These and other future technologies will continue to transform our daily lives, making our tools smarter not just for our personal use but also for the benefit of the larger community.

Rachel Berkowitz is a Corresponding Editor for Physics based in Vancouver, Canada. 